\title{
Drug design on the fly
}

\section{By Joanne Kotz, Senior Editor}

Researchers at the University of California, San Francisco and Mount Sinai School of Medicine have used a Drosophila cancer model to identify small molecule kinase inhibitors that target a discrete spectrum of kinases. ${ }^{1}$ The lead molecule blocked tumor growth in a mouse model of medullary thyroid carcinoma better than the marketed kinase inhibitor Caprelsa vandetanib. An undisclosed pharma is negotiating to license the IP.

Because multiple kinase signaling pathways are often dysregulated in the same cancer, targeting several kinases simultaneously may have greater efficacy than attacking a single kinase.

Although most kinase inhibitors in the clinic block more than one kinase, typically these molecules had been originally developed against single kinase targets; thus, any therapeutic benefits gained from hitting additional kinases is a result of chance rather than design.

"Any approach that
establishes hypotheses for
rational combinations should
be considered interesting
because determining effective
combinations preclinically
is very difficult. There is
quite some potential in this
approach. You can ask the
basic biological question
of what combinations work
best in a biological context
that is well defined-that is
powerful."
$\quad$-Christoph Lengauer,
Blueprint Medicines

inhibited RET and a variable spectrum of other kinases in a Drosophila model of a hereditary form of MTC.

The top hit from the screen, which resulted in $25 \%$ of the flies surviving to adulthood, had greater efficacy and lower toxicity than Caprelsa. In contrast, a closely related analog of the top hit inhibited RET with nearly identical potency but showed a different kinasespecificity profile and had significantly decreased efficacy.

Thus, efficacy did not correlate solely with RET inhibition.

Caprelsa is marketed by AstraZeneca plc to treat MTC. The drug was originally developed as an inhibitor of VEGF receptor (VEGFR), with weaker activity against epidermal growth factor receptor (EGFR), but later was found to inhibit RET.

The team next sought to determine which kinase targets outside of RET contributed to efficacy and toxicity.

Tests of a panel of kinase inhibitors with different levels of efficacy and toxicity based on the initial screen in genetically altered Drosophila suggested inhibiting the Drosophila homologs of RET, Src, S6 kinase (S6K), BRAF and CRAF (RAF1) contributed to efficacy, whereas blocking the homolog of mammalian target of rapamycin (mTOR; FRAP; RAFT1) led to toxicity.

Based on that information, the team designed AD80, an analog of the top hit of the original screen that had greater activity against Src, BRAF and CRAF and lower activity against mTOR.

The optimized molecule had better activity. In the MTC Drosophila model, about $80 \%$ of the flies receiving AD80 survived to adulthood.

Last, the authors tested AD80 in a mouse xenograft model of familial MTC. The molecule decreased tumor growth and weight loss, a measure of toxicity, compared with Caprelsa.

Results were published in Nature.

"Finally, we have found a screening approach that will tell us about the therapeutic index" of multitargeted kinase inhibitors, said Shokat. He noted that the key to identifying hits was starting with a small molecule library directed against RET kinase, which really "reduced the complexity."

"AD80 could not have been discovered in a cell-based screen. It is hitting multiple targets that define several key pathways," said Cagan. Identifying multitargeted molecules with "complex polypharmacology is not suited to cell-based or in vitro screens," he added.

"Any approach that establishes hypotheses for rational combinations should be considered interesting because determining effective combinations preclinically is very difficult. There is quite some potential in this approach. You can ask the basic biological question of what combinations work best in a biological context that is well defined-that is powerful," said Christoph Lengauer, CSO of Blueprint Medicines, which is developing context-specific targeted cancer therapeutics. 
The challenge now, says Lengauer, will be preclinical optimization of AD80. Lengauer said that optimizing a multitargeted kinase inhibitor may be difficult, and it might be better to use the Drosophila assay to identify a relevant set of targets that can then be inhibited therapeutically with a combination of selective inhibitors of individual kinases.

"Undesirable off-target effects can be avoided more easily when focusing medicinal chemistry efforts on tailoring compounds toward the selective inhibition of a single target rather than a desired group of proteins," he said. Lengauer noted that combining highly selective individual kinase inhibitors is the approach Blueprint intends to pursue.

Shokat countered that a single inhibitor with polypharmacology may have advantages. "My hunch is that a single compound is better because it will not give super-potent inhibition of any one target but will generally dampen the overall pathway," he said.

\section{Increasing complexity}

Shokat's team is pursuing preclinical optimization of AD80, including conducting IND-enabling studies. The researchers also plan to use the screening approach in more genetically complex cancers.

Mutant RET was the only oncogene in the current model, said Shokat. "We're really interested now in cancers that have multiple genetic alterations," he said.

Meanwhile, Cagan's laboratory is developing models of colorectal cancer. "We went into the human sequencing database and asked, what are the most common combinations of mutations reported in human colorectal cancers? The most common quadruple mutation involves Ras,
PTEN [MMAC1; TEP1], APC [adenomatous polyposis coli] and p53," he said.

Cagan's group has engineered the quadruple mutation into Drosophila models, as well as all single, double and triple combinations of those four mutations. "We built all the models in four months flat," he noted. "What we are seeing is that there is a fundamental difference between one hit or two hits compared with four hits in terms of sensitivity to drugs."

His team also is building Drosophila models of lung and breast cancer based on the genomics of patients with cancer. "We are trying to embrace the complexity, not simplify it," said Cagan.

UCSF and Mount Sinai have filed a patent application that covers composition of matter on the inhibitors identified and method of use for the Drosophila model.

Kotz, J. SciBX 5(30); doi:10.1038/scibx.2012.777

Published online Aug. 2, 2012

\section{REFERENCES}

1. Dar, A.C. et al. Nature; published online June 6, 2012; doi:10.1038/nature11127

Contact: Kevan M. Shokat, University of California, San Francisco, Calif.

e-mail: shokat@cmp.ucsf.edu

\section{COMPANIES AND INSTITUTIONS MENTIONED}

AstraZeneca plc (LSE:AZN; NYSE:AZN), London, U.K.

Blueprint Medicines, Cambridge, Mass.

Medros Inc., St. Louis, Mo.

Mount Sinai School of Medicine, New York, N.Y. University of California, San Francisco, Calif. 